\title{
APORTES DE LA CREATIVIDAD PARA EL ABORDAJE DE LA EDUCACIÓN ALIMENTARIA Y NUTRICIONAL
}

\author{
De La Cruz Sánchez, Ernesto Elías: Universidad Pedagógica Experimental \\ Libertador, Caracas (Venezuela)
}

\section{Resumen}

El propósito del presente trabajo es el de propiciar en primer lugar, un espacio para el análisis y discusión de la problemática alimentaria, aportando alternativas para su solución desde la creatividad. Y en segundo lugar, proponer técnicas creativas viables que desarrollen el potencial creativo y que permitan inducir cambios en los estilos de vida que afecten a la composición de la dieta, prevenir enfermedades y promover la salud. En el contexto de la Pedagogía, la presente propuesta esta justificada y fundamentada por dos aspectos. Por un lado en la evolución del concepto de Educar en Salud y por el otro, la oportunidad multisensorial, cognitiva, afectiva, cultural y social que ofrece el tema de los alimentos y la nutrición humana, sobre todo en la infancia. Por ello es urgente actuar en ámbitos que potencien la creatividad y que pongan en juego el logro de aprendizajes significativos y perdurables para que los niños y niñas sean capaces de adoptar estilos de vida lo mas sanos posibles, incidiendo en la información, pero también teniendo en cuenta los factores biológicos, medioambientales, psicológicos, afectivos y culturales que influyen en ellos. Se presenta un análisis de la problemática alimentaria en el contexto venezolano, una referencia a la creatividad, sus antecedentes, conceptualización y desarrollo desde la perspectiva educativa, por último se describen un conjunto de Técnicas Creativas: Mapas Mentales, Tormenta de Ideas, Analogías, Heuridramas, Relaciones Forzadas, Seis Sombreros Para Pensar, Listado de Atributos, Solución Creativa de Problemas, entre otras, partiendo de la educación alimentaría y nutricional como tema central.

Descriptores: educación alimentaria y nutricional, creatividad, técnicas creativas,

\section{Introducción}

En el contexto de la educación para la salud, la educación alimentaria y nutricional constituye una estrategia en la formación humana para la vida. Es una línea de trabajo pedagógico que nos permite adquirir mayor consciencia acerca de la importancia que damos a la primera necesidad humana y nos induce a revalorar la propia cultura alimentaria.

La alimentación y la nutrición no son sinónimas. De acuerdo a los expertos la nutrición es el conjunto de procesos bioquímicos que se producen en todos nosotros de forma individual, involuntaria e inconsciente. La alimentación en cambio, es la forma que tenemos de llevar a cabo la nutrición y que está muy influenciada tanto por la cultura, la educación y el ambiente entre otros.

Por ello, la educación en alimentación y nutrición deben orientarse a potenciar o modificar los hábitos alimentarios, involucrando a todos los miembros de la 
comunidad educativa; niños, padres, maestros y directivos. Educar sobre la necesidad e importancia de una buena alimentación implica: descubrir y erradicar creencias, mitos y conductas erróneas; promoviendo consciencia sobre las diversas funciones o roles que juega o debe jugar la alimentación en las diversas esferas de la vida, la salud, los aprendizajes, la producción, distribución y consumo de alimentos; fomentar conceptos, actitudes y conductas claras y fundamentales sobre la alimentación.

Los problemas asociados a la alimentación, a la nutrición y a la salud a nivel mundial están, arraigados en lo biológico, lo económico, lo ecológico, lo social y lo cultural; lo cual deben ser considerado como una prioridad en las políticas de desarrollo social por los diversos países (Fundación Colombiana para la Nutrición Infantil s/f). Por otra parte, las conductas en cuestiones de comida reflejan la manera de pensar sobre ella, el grupo en que se nace y se crece determinan lo que produce placer físico y psicológico, los gustos y los hábitos que se han formado en la experiencia personal e individual son inseparables. Además, toda cultura posee un bagaje de leyendas, cuentos, dichos, mitos y juegos que estructuran y dan razón a la existencia de prácticas, de prohibiciones (tabúes) y preferencias alimentarias. Iniciativas promovidas por la OMS, FAO, UCV, UCLA, LUZ, la Fundación Bengoa, el Instituto Nacional de Nutrición (INN) y las del Centro de Atención Nutricional Antímano (CANIA), nos llevan a pensar que aunque las propuestas son diversas, el consumo de alimentos en Venezuela sigue siendo un problema complejo y dinámico el cual no solo es determinado por su disponibilidad sino por variables culturales, psicológicas, ambientales y educativas.

Una de las alternativas, lo constituye su abordaje desde el punto de vista de la creatividad y del potencial creativo, desarrollado por los mismos sujetos, a través de técnicas y estrategias apropiadas, que vinculen no sólo lo cognoscitivo de la alimentación, si no aprovechen sus atributos; visuales, gustativos, olfativos, táctiles, afectivos, culturales y sociales. Esto tendrá mayor impacto en la infancia, siendo la etapa donde se fijan con mayor nitidez estos hábitos y patrones culturales, se logra el enriquecimiento de la memoria gustativa y se fortalece la identidad cultural de los venezolanos. A continuación se describen los aspectos más relevantes de la educación alimentaria y nutricional, la creatividad y su vinculación, como elementos centrales del presente trabajo y con el firme ideal de que un niño bien nutrido será un niño más creativo.

\section{La Educación Alimentaria y Nutricional en el contexto de la Educación Inicial}

En el contexto de la Educación Alimentaria y Nutricional, el ser humano recibe, desde su nacimiento y durante toda su vida, una serie de normas de conducta y refuerzos, positivos o negativos, determinantes para la toma de decisiones en la elección y consumo de sus alimentos. Adicionalmente constituye una propuesta estratégica, en la formación humana para la vida. Es una línea de trabajo pedagógico que permite adquirir mayor consciencia acerca de la importancia de la primera necesidad humana y nos induce a revalorar la propia cultura alimentaria. 
Por ello, la Educación en Alimentación y Nutrición debe orientarse a potenciar o modificar los hábitos alimentarios, involucrando a todos los miembros de la comunidad educativa; niños, padres, maestros y directivos. Educar sobre la necesidad e importancia de una buena alimentación implica: descubrir y erradicar creencias, mitos y conductas erróneas; promoviendo consciencia sobre las diversas funciones o roles que juega o debe jugar la alimentación en las diversas esferas de la vida, la salud, los aprendizajes, la producción, distribución y consumo de alimentos; fomentar conceptos, actitudes y conductas claras y fundamentales sobre la alimentación.

En la actualidad, los principales problemas nutricionales de la población mundial y venezolana se deben a dos factores fundamentales: (a) carencias que derivan en distintos grados de desnutrición; y (b) excesos o desequilibrios que se traducen en distintos trastornos. Las causas de estas caras de la realidad alimentaria son múltiples y sus efectos en la salud de la población en general impiden alcanzar los objetivos de programas educativos, científicos e industriales. En el marco de lo anterior podemos destacar que la malnutrición (desnutrición y obesidad), tiene implicaciones fundamentales en la formación integral del individuo: a) incide directamente sobre el crecimiento del sistema nervioso y sobre el funcionamiento cognitivo y por ende sobre el aprendizaje (Leiva y Col, 2001).

Razón por la cual la Educación Alimentaria y Nutricional, debe nutrir - hacer énfasisespecialmente en el nivel de Educación Inicial (primera infancia), los actores que en ella actúan; los maestros, la familia y la comunidad en general. En relación con la alimentación y específicamente a través de la absorción de los alimentos, el niño establece los primeros contactos con la realidad del mundo exterior. Comer es, además, la actividad mediante la que se desarrollan las primeras relaciones personales y en torno a las cuales el niño experimenta y vive los primeros conflictos de entendimiento con los demás y de ruptura de comunicación. Esta situación produce en ocasiones, consecuencias profundas para su socialización y en algunas circunstancias, riesgos peligrosos que es preciso prevenir.

La escuela, tiene pues, un papel fundamental a estas edades, en el aprendizaje de los buenos hábitos de alimentación. En este sentido es necesario considerar que es más fácil consolidar los hábitos saludables de manera temprana, antes que los niños y las niñas adquieran comportamientos negativos, ya que cambiar los conocimientos, las actitudes, valores y patrones de comportamiento inadecuados toma mucho más tiempo y exige también un orden o estrategia metódica, si se quiere alcanzar resultados positivos. Razón por la cual la Educación Alimentaria y Nutricional, se debe nutrir -especialmente en el nivel de Educación Inicial- de las propiedades sensoriales, del reforzamiento permanente, de las diversas realidades que confrontan los niños y niñas, de sus preferencias y desarrollo de su autonomía, que se inicia en la alimentación como el primer aprendizaje social del ser humano, del encuentro día a día con la alimentación y su potencialidad en el desarrollo de estrategias creativas que coadyuven a consolidar hábitos saludables de alimentación. 
De acuerdo con Arribas (2001), los hábitos son: (a) procedimientos de actuación mecanizados o automatizados; (b) recursos a utilizar por el docente para influir, de manera positiva en el crecimiento personal, afectivo e intelectual de sus alumnos; (c) costumbres, actitudes, formas de conducta o comportamientos las cuales conllevan pautas de conducta y aprendizajes; y (d) mecanismos estables formados por un montaje de reflejos condicionados que se encadenan entre sí. Al mismo tiempo son flexibles y posibilitan que nuestros actos se reorganicen en cada momento.

El componente educativo, que debe caracterizar a la alimentación y nutrición, se debe concebir como una estrategia de formación, lo que requiere desagregar la teoría de la nutrición, en estrategias didácticas que consideren la salud desde una visión integral y la prevención de enfermedades no transmisibles desde su consideración nutricional, ya que a través de ella se puede prevenir en salud, formar en valores y en identidad, que permitan mejorar la calidad de vida de sus ciudadanos. Una de las alternativas más viables -a nuestro buen entender- lo constituye la Creatividad.

\section{Referentes históricos acerca de la creatividad}

Los primeros acercamientos en este campo, se remontan al estudio en Ciencias Naturales a mediados del siglo XIX, con -Galton en 1869-, quién investiga la naturaleza de los genios; estudia la creatividad como fenómeno producido por la determinación hereditaria y genética, incógnita que en la actualidad es despejada solo parcialmente, pues si bien es cierto, que se estima la influencia hereditaria, no se han determinado aún sus niveles y rangos de influencia, se le considera un hecho biológico y cultural, cognitivo y afectivo, entrelazado y multivariado.

Igualmente Wallas, a principios del siglo $X X$ ofrece aportes significativos sobre el proceso creativo y la caracterización de las personas creadoras. La psicología a partir de estos intentos de acercamiento, se orienta a trabajar la creatividad desde el concepto de la imaginación creadora, es decir, independiente de la mediación genética o cultural de la creatividad, investiga la elaboración mental como factor desencadenante de procesos y productos creativos, fundados en la percepción, el procesamiento de la información y la imaginación, se enriquecen estas exploraciones investigativas con los avances en el campo de la psicometría.

A partir de allí se orienta la investigación en este campo con un énfasis empírico tratando de explicar las conductas y manifestaciones creativas. Para ello miran con especial empeño los procesos de pensamiento y elaboración mental, a la luz de los productos, los procesos y la especificidad personológica y comportamental del sujeto.

Este tipo de investigaciones le sirvieron de base a la comunidad internacional, para dirigir sus miradas a procesos, con marcadas tendencias estructurales y funcionales, a centrar los intereses en las observaciones y comportamientos de los sujetos, particularmente el de los inventores como fuente de estudio y acercamiento al hecho creativo, igualmente en los procesos de construcción del conocimiento mediado por las acciones de formación y aprendizaje; exploraciones en las cuales se demuestra la contribución de la creatividad en el éxito escolar y ganancias significativas a través de educación en creatividad. 
En la década de los 60, el referente creativista llega a Europa; pioneros como Handin, Beudot, Kaufman, la proyectan y la difunden, se destaca en el campo educativo los aportes 1970-1980, de R. Marín; Saturnino De la Torre, D. de Prado, para citar solo algunos, quienes consolidan una comunidad académica en el área en el territorio europeo. En la década del 70 y 80, llega a América Central y Suramérica, con mención de los desarrollos investigativos de Mauro Rodríguez (México), Margarita de Sánchez y Antonio Machado (Venezuela), A. Galeano, G. Aldana, C. Vasco (Colombia), A. Mitjáns (Cuba) M. Max Neef. (Chile), entre otros.

Desde la perspectiva pedagógica, De La Torre (1997), plantea que la formación para el futuro de un ser innovador y creativo se inscriben en cuatro dimensiones producto del juego del ser, saber, hacer y querer, en el cual la combinación del ser y el hacer proporcionan la autoafirmación y el desarrollo de las habilidades personales y sociales; la combinación del ser y el querer, determinan la formación para el cambio y la creatividad; la combinación del hacer y el saber, ofrece la dotación instrumental y de estrategias cognoscitivas; y la combinación del querer y el saber, nos proporcionan las competencias profesionales y direccionamientos; nuestra formación ha tendido a enfatizar el saber y el hacer y desde luego el saber hacer, pero es necesario fortalecer igualmente los procesos de desarrollo de la dimensión afectiva y volitiva, es decir, favorecer los campos de la afectividad, la inteligencia emocional y la voluntad. Es en general en la capacidad de asombro y la motivación intrínseca donde se nace la creatividad, es generalmente en el emprendimiento y en la determinación donde se forja y finalmente es en la imaginación y la divergencia donde aflora. Una educación integral y una estrategia creativa es el mejor camino de encuentro para la creación desde los primeros años de vida del niño y la niña.

Estos, en resumen y a rasgos generales, son algunos de los puntos de vista sobre la dimensión del pensamiento creativo que han venido trabajando los investigadores en creatividad desde diversas, ópticas, lógicas e intereses; se aprecian tendencias biologicistas, cognitivistas, culturalistas, humanistas, transpersonales y existencialistas de la creatividad; también es un campo abierto a los modos de hacer ciencia, bien sean, explicativos, interpretativos o dirigidos a la acción misma. Pasemos a esbozar algunas precisiones sobre la creatividad.

\section{Concepciones acerca de la creatividad}

La primera apreciación, es la creatividad como condición humana a ser, querer, saber y hacer, para descubrir, redefinir y recrear la naturaleza; definición acuñada fruto de un interesante seguimiento de causa y efecto. En este sentido se recogería la apreciación de la creatividad como un acto de expresión, reconocimiento y trascendencia, que en términos de Aldana (1995), se define como una manera especial de pensar, sentir y actuar, que conduce a un logro o producto original, funcional y estético, bien sea para el propio individuo o para el grupo social al que pertenece. Aplicadamente se interpretaría en su proceso como; la combinación de ideas nuevas o existentes para formar una nueva, que satisface mejor una necesidad.

La creatividad por su condición generadora, es necesario expresarla en términos de algo patente, visible, para así identificarla y entenderla; es por esto que la mayoría de sus definiciones apuntan más a sus resultados que a la esencia misma del acto 
creador, Parnes, (citado en Paredes 2003), plantea la creatividad como acción para la creación de ideas nuevas y valiosas. El programa PRYCREA de la Habana, considera la creatividad como el pensamiento de más alto orden y lo define como la potencialidad transformativa cognitivo-afectiva, caracterizada por la generación, la expresión, la flexibilidad y la autonomía (González 1996).

De acuerdo con Paredes (2003), la creatividad es el proceso de presentar un problema a la mente con claridad (ya sea imaginándolo, visualizándolo, suponiéndolo, meditando, contemplando, etc.) y luego originar o inventar una idea, concepto, noción o esquema según líneas nuevas o no convencionales, supone estudio y reflexión más que acción. Desde esta perspectiva la creatividad es la capacidad de ver nuevas posibilidades y hacer algo al respecto. Cuando una persona va más allá del análisis de un problema e intenta poner en práctica una solución se produce un cambio.

Por otra parte, la creatividad según Penagos (2003), es la generación de productos, cog-mociones y/o conductas relevantes para una situación de destreza o conocimiento insuficiente. Vista así, es entonces un fenómeno, multivariable en su propia definición por lo que es necesario abordarla con enfoques poderosos que tengan también una naturaleza multivarible. 


\section{Cuadro 1}

Principales referentes a la conceptualización de la creatividad

\begin{tabular}{ll}
\hline Autor & \multicolumn{1}{c}{ Concepto de Creatividad } \\
\hline Weithemer(1945) & $\begin{array}{l}\text { El pensamiento productivo consiste en observar y tener en cuenta rasgos y } \\
\text { exigencias estructurales. Es la visión de verdad estructural, no fragmentada. }\end{array}$ \\
Guilford (1952) & $\begin{array}{l}\text { La creatividad, en sentido limitado, se refiere a las aptitudes que son } \\
\text { características de los individuos creadores, como la fluidez, la flexibilidad, la } \\
\text { originalidad y el pensamiento divergente". }\end{array}$
\end{tabular}

Osborn (1953) Aptitud para representar, prever y producir. Conversión de elementos conocidos en algo nuevo, gracias a una imaginación poderosa

Fromm (1959) La creatividad no es una cualidad de la que estén dotados los artistas y genios, sino actitud que pueden poseer cada persona.

Rogers (1959) La creatividad es una emergencia en acción de un producto relacional nuevo, manifestándose por un lado la unicidad del individuo y por otro los materiales, hechos, gente o circunstancias de su vida.

Donald La creatividad consiste en un proceso que cumple al menos tres condiciones: MacKinnon Implica una idea o respuesta nueva. La idea debe adaptarse a la realidad, (1960) solucionar un problema o alcanzar una meta. Es preciso que se mantenga la idea original, se evalúe y desarrolle hasta su aplicación final.

Getzels y La creatividad es la habilidad de producir formas nuevas y reestructurar Jackson (1962) situaciones estereotipadas.

Parnes (1962) Capacidad para encontrar relaciones entre ideas antes no relacionadas y que se manifiestan en forma de nuevos esquemas o productos.

Ausubel (1963) La personalidad creadora es aquella que distingue a un individuo por la calidad y originalidad fuera de lo común de sus aportaciones a la ciencia, al arte, a la política.

Piaget (1964) La creatividad constituye la forma final del juego simbólico de los niños, cuando, éste es asimilado en su pensamiento.

Torrance (1965) Es un proceso que vuelve a alguien sensible a los problemas, deficiencias, o lagunas en los conocimientos y lo lleva a identificar dificultades, buscar soluciones, o formular hipótesis, aprobar y comprobar estas hipótesis, además de comunicar los resultados.

Guilford (1971) Capacidad o aptitud para generar alternativas a partir de una información dada poniendo el énfasis en la variedad, cantidad y relevancia de los resultados

De Bono (1974) Es una aptitud mental y una técnica de pensamiento.

David Perkins Conjunto de capacidades y disposiciones que hacen que una persona produzca (1981) con frecuencia productos creativos es decir: originales, adecuados a la realidad, complejos, trascendente, unitarios y simbólicamente significativos.

Richard Mayer Actividad cognitiva que tiene como resultado soluciones nuevas a un problema. (1983) Enseñar creatividad implica enseñar a las personas como generar nuevas ideas para resolver una situación dada.

Teresa Amabile La creatividad se conceptualiza mejor no como un rasgo de la personalidad, ni (1983) como una habilidad general, sino como la conducta resultante de una constelación particular de características personales, habilidades cognitivas e influencias ambientales. Esta conducta, que se pone de manifiesto en productos o respuestas, sólo se puede explicar de una forma completa mediante un modelo que abarque estos tres conjuntos de factores.

Bandura(1987) Una síntesis innovadora de influencias captadas de distintas fuentes $\mathrm{y} / \mathrm{o}$ modelos.

Fuente: Definiciones de Creatividad aportadas por: Esquivas (2003), Huidobro (2002), De La Torre (1993). 
Cuadro 1. Cont.

$\begin{array}{ll}\text { El pensamiento creativo puede ser definido como un proceso metacognitivo de } \\ \text { Pesut (1990) } & \begin{array}{l}\text { autorregulación, en el sentido de la habilidad humana para modificar } \\ \text { voluntariamente su actividad psicológica propia y su conducta o proceso de } \\ \text { automonitoreo. }\end{array}\end{array}$

De La Torre Capacidad y actitud para generar ideas nuevas y comunicarlas

(1991)

Mintzberg

(1991)

Mitjans (1995)

La creatividad consiste en el acoplamiento y el equilibrio entre el análisis y la intuición.

Creatividad es el proceso de descubrimiento o producción de algo nuevo que cumple con las exigencias e una determinada situación social, proceso que, además tiene un carácter personológico.

Csikszenmialyi La creatividad es cualquier acto, idea o producto que cambia un campo ya (1996)

Esquivas

(1997) existente, o que transforma un campo ya existente en algo nuevo

La creatividad es un proceso mental complejo, el cual supone: actitudes, experiencias, combinatoria, original y juego, para lograr una producción o aportación diferente a lo que ya existía.

Sternberg

(1998)

Rodríguez

(1999)

Gardner (1993)

La creatividad es un fenómeno de múltiples facetas, tres de las cuales resultan críticas: la inteligencia, el estilo intelectual y la personalidad.

La creatividad es la capacidad de producir cosas nuevas y valiosas.

El individuo creativo es la persona que resuelve problemas con regularidad, elabora productos o define cuestiones nuevas en un campo, de un modo que al principio es considerado nuevo, pero al final llega a ser aceptado en un contexto cultural complejo.

Gagne (1998) La creatividad puede ser considerada una forma de solucionar problemas, mediante intuiciones o una combinación de ideas de campos muy diferentes de conocimientos.

Grinberg Capacidad del cerebro para llegar a conclusiones nuevas y resolver problemas en (1999) una forma original. Se relaciona con la efectiva integración de ambos hemisferios.

Fuente: Definiciones de Creatividad aportadas por: Esquivas (2003), Huidrobo (2002), De la Torre (1993).

Como se observa en dicho cuadro, el concepto de la creatividad se encuentra en una situación compleja, polisémica, en la que su definición y su praxis se vuelven confusas por los límites mínimos existentes entre la creatividad y la inteligencia, en los cuales se pueden establecer y precisar diferencias, más no dicotomías. Podríamos intentar ver la creatividad desde un plano diferente al de la lógica y al de la racionalidad, para poder introducir el concepto de la creatividad en un contexto que le permita al ser humano, en sus actos de creación, poder representar el mundo de muchas formas nuevas y sorprendentes, aunque éstas no sean comprendidas y predecibles como lo exige el discurso formal de las ciencias La creatividad es un sendero abierto a la incertidumbre, al azar, al desorden, al caos y a las diferentes brechas de nuestros mapas mentales, lo que hace difícil su predicción en el tiempo y en el espacio. Un acto creativo no es un solo camino, son muchos caminos o planos impredecibles de mentes humanas preparadas por la cultura o por la ciencia (Jiménez 1998). 
Las concepciones presentadas constituyen una aproximación al entendimiento del desarrollo creativo, intentan ser el inicio de una discusión más amplia en donde se estudien las condiciones de la creatividad y su relación con el desarrollo de la conciencia. Es por ello que la educación tiene, hoy más que nunca, la tarea de liberar la creatividad que nace con cada niño y niña, de crear las oportunidades para su desarrollo y de encaminarlo para el bien personal y social. Desde la perspectiva de De La Torre (1997, p. 19), la creatividad es un fenómeno que se mueve entre atributos personales y las exigencias sociales, porque en último término es la sociedad la que promueve y sanciona el valor o relevancia de las actividades creativas. Así mismo, la creatividad es fruto de la interacción sociocultural y sólo será plena cuando engendre mejoras sociales o culturales y al igual que la educación o la salud, son bienes y exigencias sociales. La creatividad como bien social se relaciona con sus implicaciones en el desarrollo humano, en el desarrollo científico y cultural y como concepto clave de conciencia al servicio de la comunidad. Una conciencia colectiva propicia a la creatividad es aquella que promueve la tolerancia, valora la independencia de pensamiento, presta atención a la diversidad, reconoce el esfuerzo, premia la iniciativa, valora positivamente las nuevas ideas, etc (op. cit. p. 71). El fenómeno de lo creativo ha de ser encarado desde diversas orientaciones y de modo interdisciplinar. La creatividad extiende sus ramificaciones al ámbito neurobiológico, psicológico, organizativo y muy particularmente pedagógico. En todo caso y en líneas generales las diversas investigaciones sobre el tema vienen planteando la creatividad desde cuatro dimensiones básicas: personal, proceso, medio y producto, mostrándose en éste un abanico de manifestaciones tan variado como actividades humanas (De La Torre, 1997, p. 57). Lo cual se puede aprecia en el gráfico № 1.

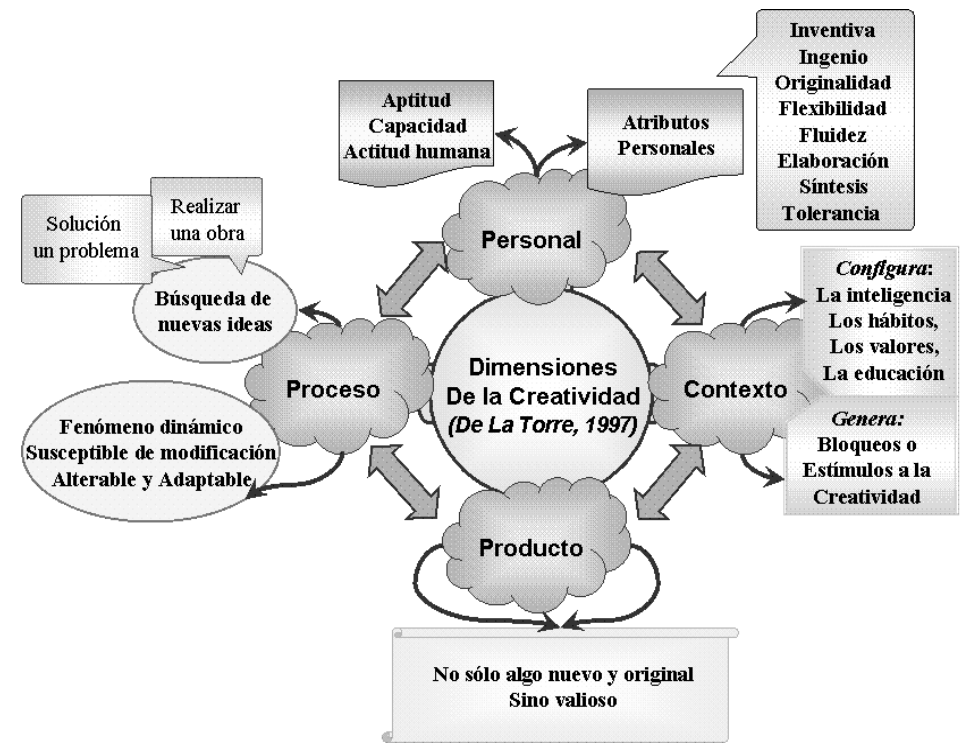

Gráfico № 1. Dimensiones de la Creatividad. De La Torre (1997). 


\section{La Creatividad en Educación}

Numerosos estudios destacan el papel del maestro, tanto como elemento facilitador del desarrollo y de la expresión de la creatividad, como elemento bloqueador de las posibilidades de crecimiento del alumno. Como es del conocimiento general, el maestro puede por un lado despertar el interés del alumno sobre un asunto o área de conocimiento y por otro lado llevarlo a odiar una determinada materia. Puede concientizar al alumno de sus talentos y posibilidades, o minar su confianza sobre su propia capacidad y competencia. Puede contribuir tanto para la formación de un autoconcepto positivo, como por el contrario, para la formación de una imagen negativa de si mismo, llevando al alumno a cerrarse sobre los recursos de su imaginación y capacidad de crear Soriano (2003). Según esta autora, educar en la creatividad es educar para el cambio y formar personas ricas en originalidad, flexibilidad, visión futura, iniciativa, confianza, amantes de los riesgos y listas para afrontar los obstáculos y problemas que se les van presentado en su vida escolar y cotidiana, además de ofrecerles herramientas para la innovación. La creatividad puede ser desarrollada a través del proceso educativo, favoreciendo potencialidades y consiguiendo una mejor utilización de los recursos individuales y grupales dentro del proceso de enseñanza-aprendizaje. Siguiendo con estas ideas no podríamos hablar de una educación creativa sin mencionar la importancia de una atmósfera creativa que propicie el pensar en el salón de clase. La concepción acerca de una educación creativa parte del planteamiento de que la creatividad está ligada a todos los ámbitos de la actividad humana y es el producto de un devenir histórico social determinada, por ello educar en la creatividad implica partir de la idea de que ésta no se enseña de manera directa, sino que se propicia y que para esto es necesario tomar en cuenta las sugerencias presentadas en el siguiente gráfico.

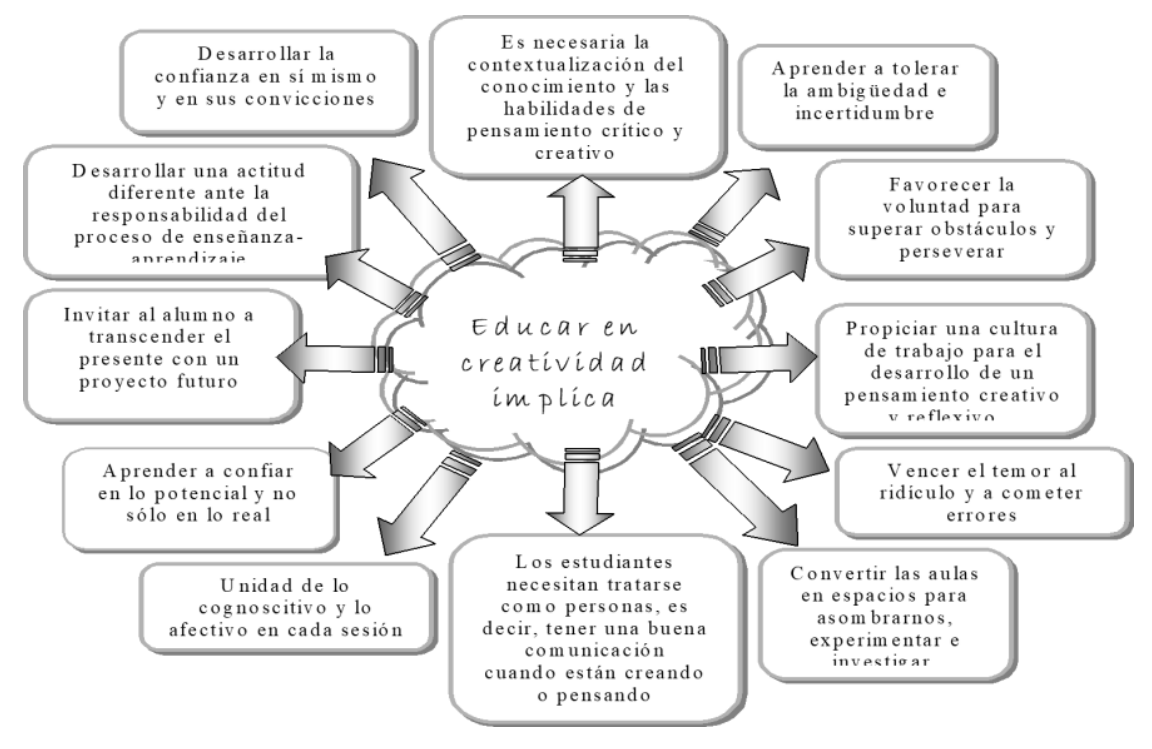

Grafico № 2. Implicaciones de la creatividad, basado en Soriano (2003).

Siguiendo con esta manera de pensar tendríamos un concepto de creatividad acorde con los planteamientos anteriores, que bien puede ser el siguiente. Creatividad es el 
potencial humano integrado por componentes cognoscitivos, afectivos, intelectuales y volitivos, que a través de una atmósfera creativa se pone de manifiesto, para generar productos novedosos y de gran valor social y comunicarlos transcendiendo en determinados momentos el contexto histórico social en el que se vive. Podemos afirmar, que una educación creativa es una educación en la cual no solamente resulta valioso el aprendizaje de nuevas habilidades y estrategias de trabajo, sino también el desaprendizaje de una serie de actitudes que en determinados momentos nos llenan de limitaciones para ser creativos o para permitir que otros lo sean.

A manera de conclusión pudiéramos señalar que Educar en la Creatividad debe orientarse al desarrollo personal y mejora profesional de la práctica educativa de todos los implicados en el proceso de enseñanza y de aprendizaje, dentro de un contexto histórico social dirigido a la integración educativa, partiendo del criterio de que la creatividad nos permite tener una actitud flexible y transformadora que propone romper las murallas o barreras para edificar la nueva escuela del futuro, cuyos principales apellidos sean: integrada, solidaria, respetuosa, reflexiva, divergente, desarrolladora, abierta y consistente con las necesidades de todos los alumnos.

Todo lo anteriormente expresado conlleva a una educación en la creatividad que propicie un sistema de actividades y comunicación donde el pensamiento reflexivo y el creativo se desarrollen a la par de una actitud coherente. Cada alumno que egrese de una escuela formado con esta visión deseará que en los lugares donde llegue se manifieste el pensar, crear, sentir, comunicar y compartir por respeto a sí mismo y a sus semejantes, De la Torre (1995).

De los trabajos consultados sobre la creatividad y la educación, podemos concluir que existe un consenso acerca de la viabilidad y conveniencia de estimular las diferentes habilidades que constituyen el pensamiento creativo, el cual condensa muchas de las posibilidades propuestas para adaptarse efectiva y oportunamente al cambio. A continuación se presenta un conjunto de técnicas creativas, a manera de propuestas, las cuales se ejemplifican con aspectos, temas y problemas vinculados al campo de la educación alimentaria y nutricional.

\section{Técnicas Creativas aplicables a la Educación Alimentaria y Nutricional}

\section{- Los Mapas Mentales}

Es una técnica de usos múltiples. Su principal aplicación en el proceso creativo es la exploración del problema y la generación de ideas. En la exploración del problema es recomendable su uso para tener distintas perspectivas del mismo y desarrollar diversas formas de abordarlo. Ejemplo: a) Estudio de los hábitos alimentarios, b) el problema de la obesidad infantil, c) los medios de comunicación, d) el consumo de frutas y vegetales, entre otros.

\section{- BRAINSTORMING (Tormenta De Ideas)}

El Brainstorming, también llamado torbellino de ideas, tormenta de ideas, lluvia de ideas, es una técnica eminentemente grupal para la generación de ideas en la cual las mismas se van generando por el grupo, analizando y aportando en forma individual y grupal. Ejemplo: ¿Qué podemos hacer para solucionar el hábito inadecuado de consumo de chucherías, bebidas gaseosas y golosinas?, ¿Qué 
podemos proponer para mejorar la calidad de los alimentos de la cantina escolar? ¿Qué alternativas saludables podemos incorporar en nuestro desayuno?

\section{- Listado De Atributos}

Es una técnica creada por R.P. Crawford, ideal para la generación de nuevos productos. También puede ser usada en la mejora de servicios o utilidades de productos ya existentes. Para que esta técnica dé resultados, primero se debe realizar un listado de las características o de los atributos del producto o servicio que se quiere mejorar para, posteriormente, explorar nuevas vías que permitan cambiar la función o mejorar cada uno de esos atributos. Ejemplo: Elaboración y conservación de jugos naturales, postres, yogur, basados en frutas y vegetales de temporada. Estudio e incorporación de la tradición local o regional en dichas preparaciones.

\section{- Seis Sombreros Para Pensar}

Es una técnica creada por Edward De Bono, una herramienta de comunicación utilizada en todo el mundo para facilitar la resolución o el análisis de problemas desde distintos puntos de vista o perspectivas. Se trata de un marco de referencia para el pensamiento que puede incorporar el pensamiento lateral. El autor nos propone un método que nos permite pensar de manera más eficaz. Los seis sombreros representan seis maneras de pensar y deben ser considerados como direcciones de pensamiento más que como etiquetas para el pensamiento, es decir, que los sombreros se utilizan proactivamente y no reactivamente. Los beneficios derivados del uso de esta técnica son tres: a) Fomenta el pensamiento paralelo, b) Fomenta el pensamiento en toda su amplitud, y c) Separa el ego del desempeño. Ejemplo: ¿El valor o importancia del alimento para la conservación de la salud? ¿La idea del concepto de salud desde la perspectiva curativa vs perspectiva preventiva? ¿Valor de la educación en la formación de hábitos saludables? ¿la tradiciones culturales en la promoción de la salud infantil?

\section{- $D O I T$}

Do It, traducido "hágalo" se basa en los siguientes conceptos: Definir, Abierto, Identificar y Transformar. Esto viene a significar la necesidad de definir problemas, abrirse a muchas soluciones posibles, identificar la mejor solución y luego transformarlo en acción con eficacia. Ejemplo: a) la venta de alimentos poco adecuados en la cantina escolar, o las ventas ambulantes de alimentos; b) La importancia de la higiene y conservación de los alimentos que consumimos; c) las enfermedades transmitidas por alimentos contaminados; d) efectos de las comidas rápidas o chatarras en aspectos como la salud, nuestra cultura, la economía regional; f) la presencia de pesticidas en los alimentos y su efecto en la salud.

\section{- Método 635}

Seis personas, se reúnen alrededor de una mesa para generar ideas relativas a un tema previamente planteado. Se da a cada una de ellas una hoja en blanco. Tres ideas, son las que tendrá que escribir cada participante en su hoja, de manera concisa y breve ya que sólo dispone de cinco minutos para escribirlas; un vez transcurridos, cada uno pasará su hoja al compañero de al lado y se repetirá el proceso de escribir tres nuevas ideas en otros cinco minutos, después de haber 
leído las ideas de los participantes anteriores, que servirán a su vez como fuente de nueva inspiración. Al completar el ciclo de seis intervenciones de cinco minutos, en el que se habrán hecho circular todas las hojas, ordenadamente y una sola vez para cada uno de los participantes, se podrá disponer de dieciocho ideas en cada hoja, lo que puede suponer ciento ocho ideas en sólo media hora. Habrá algunas repetidas, sobre todo al principio de cada hoja y unas cuantas absurdas. Ejemplo: Permitir conocer aspectos de su alimentación, el valor que le representan los alimentos, su conocimiento y percepción de los alimentos, la calidad y cantidad de los alimentos que consume a fin de determinar y proponer alternativas para elevar su calidad y mejorar sus hábitos alimentarios. También podrá detectar los efectos del consumo de alimentos poco saludables en comparación con alimentos adecuados que aporten sus necesidades diarias de nutrientes y energía.

\section{- Relaciones Forzadas}

Método creativo desarrollado por Charles S. Whiting en 1958. Su utilidad nace de un principio: combinar lo conocido con lo desconocido fuerza una nueva situación. De ahí pueden surgir ideas originales. Es muy útil para generar ideas que complementan al Brainstorming cuando ya parece que el proceso se estanca. Ejemplo: a) preparar combinaciones con alimentos poco conocidos por el grupo, b) estudio de cómo las poblaciones en diferentes épocas y regiones del país abordaron los problemas de adquisición y consumo de alimentos, c) simulación hipotética de situaciones de riesgo o no prevista y que exista la carencia de algún alimento.

\section{- Analogías}

Consiste en resolver un problema mediante un rodeo: en vez de atacarlo de frente se compara ese problema o situación con otra cosa. Gordon, creador de la Sinéctica (método creativo basado en el uso de las analogías) insistía en que "se trata de poner en paralelo mediante este mecanismo unos hechos, unos conocimientos 0 unas disciplinas distintas". Por ejemplo, un problema empresarial lo intentamos resolver buscando algún problema análogo en otras disciplinas: en la biología, en la historia, en un deporte colectivo. Ejemplo: a) desarrollo de campañas publicitarias para la promoción de algún alimento producto elaborado por el grupo, b) experiencias científicas utilizando los alimentos para comprender otros conceptos como: concentración, Oxidación, Solubilidad, Calor, Estados de la Materia, Separación de Mezclas, entre otras.

- Solución Creativa De Problemas En Grupo.

CPS son las siglas de Creative Problem Solving, un método para la solución creativa de problemas desarrollado inicialmente por Alex Osborn y después complementado por Sidney Parnes. Ellos fueron los que crearon la Escuela de Buffalo (USA). Este método nos ofrece "un esquema organizado para usar unas técnicas específicas de pensamiento crítico y creativo" con vistas al logro de resultados novedosos y útiles. (Isaksen). Ejemplo: Análisis de las enfermedades no transmisibles y su relación con los hábitos alimentarios, el sedentarismo, la obesidad, la anorexia y la bulimia, aspectos psicológicos de los alimentos a diferentes edades y su visión desde la perspectiva femenina y masculina.

\section{- Técnica Clásica}


Según James Webb Young esta técnica se desarrolla en cinco fases en un orden determinado; ninguna de ellas puede darse antes de que la precedente haya quedado concluida: a) recogida de la materia prima o información relacionada con el problema, b) Trabajo de las ideas recopiladas. Masticación de la información, c) Incubación inconsciente, d) Inspiración o surgimiento de la idea, e) Configuración final y desarrollo de la idea para su utilización práctica. Estas etapas reflejan el proceso completo a través del cual se producen las ideas. Este proceso es una técnica operativa que puede ser aprendida y controlada. Ejemplo: ¿Alternativas a la transición alimentaria? ¿Análisis de la problemática alimentaria desde diferentes perspectivas y su proyección a escenarios futuros?

- El pensamiento mediante imágenes: la visualización

La característica básica de esta técnica es la preponderancia de su no verbalidad. La utilización de este método se basa en la idea de que el lenguaje estructura el pensamiento de una forma lógica para que sea posible la comunicación. Esto produce un control del pensamiento por su parte consciente. Pero si se da prioridad a la parte no verbal o visual sobre la verbal, se consigue mayor rapidez y versatilidad de éste.

Para que le sea más fácil realizarlo por primera vez. Es muy importante que intente visualizar, es decir, representar la imagen del objeto en la mente y conservarla durante todo el tiempo de realización. También es importante que sólo piense en imágenes y sensaciones. Déjelas que fluyan, no utilice su lenguaje interno. Vista, Oído, Olfato, Gusto Tacto. Ejemplo: a) Representaciones empleando los alimentos como fuente de ideas, b) Asociaciones de alimentos con imágenes, sonidos, sensaciones táctiles y gustativas, compartiendo y respetando las diferencias individuales

\section{- Adividanzas}

Esta actividad consiste en propiciar el desarrollo y creación de adivinanzas basadas en características, funciones, sabores, dibujos de objetos. Ejemplo: a) Características de las frutas y vegetales en su estado natural, b) Preparación de jaleas con base a los alimentos suprimiendo alguno de los sentidos como la vista y el olfato, c) Nombre y características de los frutos y vegetales en diferentes regiones.

\section{- Heuridrama}

Es el descubrimiento a través de la acción dramática. El soporte y fundamento metodológico radica en la identificación con el problema u objeto de estudio, vivenciándolo y expresándose desde él. El tipo de analogías o identificaciones puede ser muy variado. Ejemplo: El diseño de una merienda, donde los alumnos planifiquen desde la selección del menú, la compra, la preparación, y el consumo en el aula, representando los diferentes roles de la familia. Esto permitirá fortalecer la comunicación, la organización y el valor de compartir los alimentos con el grupo.

\section{Consideraciones finales}

Debemos considerar que el principal enemigo de la salud urbana es el estilo de vida lo que incluye a los hábitos alimentarios, lo cual no debe ser considerado como un 
asunto individual sino colectivo y que en nuestro país se balancea entre la abundancia y la escasez (desnutrición y obesidad), siendo el sedentarismo una problemática importante en el bienestar físico.

La educación alimentaria, sin ser la respuesta al problema, ha demostrado a través de múltiples experiencias, ser una alternativa viable para lograr cambios positivos en las conductas alimentarias, las cuales se establecen en los primeros años de vida.

Por otra parte, la creatividad es una condición necesaria para el crecimiento de un país, para el desarrollo de la humanidad, para la calidad de lo humano. Empezar y fomentar acciones que contribuyan a la investigación, al desarrollo de la creatividad, es urgente e indispensable.

Las estrategias destacadas aquí, son instrumentos del pensar y aprender, y cuantos más instrumentos se puedan utilizar hábilmente, mayor será el éxito que experimenten nuestros estudiantes ante una variedad de actitudes o problemas presentados. También deben emplearse para superar bloqueos del pensamiento creativo impuestos por prácticas educacionales, culturales, emocionales y ambientes hostiles conformistas y estandarizadas, deben abocarse a estimular la producción de nuevas ideas para solucionar problemas y enfrentar los nuevos desafíos.

Por ello es urgente actuar en ámbitos que potencien la creatividad y que pongan en juego el logro de aprendizajes significativos y perdurables; lo fundamental es la manera en que se lleva a cabo el proceso creativo tanto como el producto que se logra, porque este último es consecuencia de aquel.

La creatividad humana se conjuga en la mezcla exacta del contenido que aporta el campo imaginario y lo racional, realiza una síntesis entre ambos. El sujeto opera con: mente, cuerpo; interrelaciona e interactúa con el medio ambiente dentro de una situación espacio-temporal, por la cual pone en juego el pensamiento, los sentidos, la acción, su íntima vinculación; nada mas pertinente para ello, que el abordaje de la educación alimentaria y nutricional como campo experiencial por toda la vida.

\section{Referencias bibliográficas}

ALDANA, G. (1995). Creatividad, Formación e Investigación. Misión Ciencia. Educación y Desarrollo. Fuentes Contemporáneas I. Tomo 5. Colección documentos de la Misión Ciencia. Ediciones de la Presidencia de la República. Concedería presidencial para el desarrollo Institucional. Conciencia. Bogota. D.C.

ARRIBAS, LLeixa (2001). La Educación Infantil de 0-6 años. Ediciones Paidotribo. Barcelona-España.

DE LA TORRE, S. (1993). Creatividad Plural. Sendas para indagar sus múltiples perspectivas. Ediciones Promoción y Publicaciones Universitarias. Barcelona. España.

DE LA TORRE, S. (1995). Creatividad Aplicada. Recursos para una Formación Creativa. España: Escuela Española. 
DE LA TORRE, S. (1997). Creatividad y Formación. Identificación, Diseño y evaluación. México: Trillas.

ESQUIVAS, M. (2003). Creatividad, definiciones, antecedentes y aportaciones. Rev. UNAM. 5 (1). p. 35-67. Documento en Línea. Disponible: [http://www.revista.unam.mx/vol.5/num1/art4/portada.htm\#]. (Consulta: 2003, Abril, 15)

Fundación Colombiana para la Nutrición Infantil (s/f.) La alimentación y nutrición infantil [Documento en línea]. Disponible: [http://www.coalimentación.com/?page id=36] [Consulta: 2003, Agosto 11].

GONZÁLEZ C. (1996) Programa Estratégico de Gestión Creativa. Universidad Nacional de Colombia. Fuente [www.unac.gov.col/] (consulta 25-09-2003)

HUIDOBRO, T. (2002). Una definición de la Creatividad a través del estudio de 24 autores seleccionados. Memoria para optar al grado de Doctor. Universidad Complutense de Madrid. [Documento en línea]. Disponible: [http://eprints.ucm.es/tesis/psi/ucm-t25705.pdf.] (Consulta: 2003, Agosto 11]

JIMÉNEZ, C. (1998). Pedagogía de la Creatividad y de la Lúdica, Emociones, Inteligencia y Habilidades secretas. Editorial Mesa Redonda. Bogotá.

LEIVA PLAZA, Boris, Nelida Inzunza Brito, Hernán Pérez Torrejón, Veronica Castro Gloor, Joan Manuel Janzana Medina, Triana Toro Díaz, Arturo Navarra Díaz, Atilio Almagiá Flores, Arturo Navarro Díaz, María Soledad Urrutia Cáceres, Jorge Cervilla Oltremari y Daniza Ivanovic Marincovich. (2001). Algunas consideraciones sobre el impacto de la desnutrición en el desarrollo cerebral, inteligencia y rendimiento escolar. . ALAN. vol.51, no.1, p.64-71. Disponible [http://www.scielo.org.ve/scielo.php?script=sci arttext\&pid=S0004-0622200 1000100009\&lng=es\&nrm=iso>. ISSN 0004-0622]. (Consulta 11/02/2003).

PAREDES AGUIRRE, Alfonso (2003). Creatividad. Documento en línea. Disponible: [http://sardis.upeu. edu.pe/ alfpa/creatividad.htm] (Consulta 20-09-2003).

PENAGOS C. Julio C. (2003) Creatividad y Modelo Holodimérgico, Explorando algunas dimensiones del modelo holodimérgico Trabajo presentado en el IV Coloquio de Psicología Transpersonal en la Universidad de las Américas, Puebla. Fuente: [http://homepage.mac.com/penagoscorzo/index.htm] (consulta 15-11-2003)

SORIANO DE ALENCAR E. (2003) La escuela y el desarrollo del talento creativo. Innovando, Año 2 № 8 Enero 2003, p.25-37. 